NOTAS Y EXPERIENCIAS / NOTES AND EXPERIENCES

\title{
La información y documentación científica en el CSIC
}

\author{
Luis M. Plaza* \\ *Consejo Superior de Investigaciones Científicas (CSIC), Madrid, Spain. \\ Correo-e: luis.plaza@csic.es
}

Recibido: 22-10-2013; 2a version: 20-11-2013; Aceptado: 04-12-2013.

Cómo citar este artículo/Citation: Plaza, L.M. (2014). La información y documentación científica en el CSIC. Revista Española de Documentación Científica, 37(2):e048. doi: http://dx.doi.org/10.3989/redc.2014.2.1129

Resumen: Esta contribución pone de relieve la importancia estratégica del ámbito disciplinar de la Información y Documentación Científica y, tras una resumida panorámica de su desarrollo en el contexto del Consejo Superior de Investigaciones Científicas (CSIC), se argumenta la necesidad de dotar a la institución con una Política de Información Científica, al amparo de la cual generar un Sistema Integrado de Información y Documentación capaz de contribuir a la misión y objetivos generales del CSIC y de servir de referente en el marco del actual sistema público español de I+D.

Palabras clave: Información y documentación científica; política científica; política de información científica; sistemas de información y documentación; CSIC; España.

\section{Information Science in the CSIC}

Abstract: This contribution highlights the strategic importance of the disciplinary field of Documentation and Information Science. After a summary overview of its development in the context of the Spanish National Research Council (CSIC), the author argues the need for an institutional Scientific Information Policy, under which to generate an Integrated System for Information and Documentation capable of contributing to the mission and objectives of the CSIC and of serving as a reference in the context of the current Spanish public R\&D system.

Keywords: Information science; documentation; research policy; scientific information policy; research information systems; CSIC, Spain.

Copyright: (c) 2014 CSIC. Este es un artículo de acceso abierto distribuido bajo los términos de la licencia Creative Commons Attribution-Non Commercial (by-nc) Spain 3.0. 


\section{INTRODUCCIÓN}

La Información y Documentación Científica es un ámbito disciplinar de enorme importancia estratégica para cualquier institución científica de relevancia, como es el caso del Consejo Superior de Investigaciones Científicas (CSIC), o de cualquier otra entidad de I+D que base su actividad en criterios de excelencia y que tenga una proyección internacional (Zins, 2007).

En el CSIC, este ámbito disciplinar se ha ido conformando mediante la creación de lo que podríamos entender como dos ejes fundamentales; por una parte, el constituido por los Institutos y Centros de Información y Documentación propiamente dichos y, por otra, por la Red de Bibliotecas de la institución. A las importantes labores desempeñadas a lo largo de los años por estas entidades, hay que sumar las correspondientes al Departamento de Publicaciones y al imprescindible apoyo prestado a todas ellas por las unidades centralizadas de informática del CSIC.

Si bien algunas definiciones de amplia aceptación internacional equiparan la expresión "Información y documentación científica" a la expresión inglesa "Information Science", éstas incluyen los ámbitos de actividad propios, por una parte de documentalistas $y$, por otra, de bibliotecarios y archiveros. Pese a las estrechas relaciones entre uno y otro ámbito, el de información y documentación científica es el que incluye un mayor abanico de actividades, entre las que destacan las tareas de análisis de la información, que a su vez conlleva diversas aplicaciones tanto en el ámbito de los servicios y productos de información científica como en el contexto de la investigación sobre las dinámicas de la ciencia y la tecnología. En base a su incuestionable importancia estratégica, este artículo aborda de forma específica la evolución de la información y documentación científica en el CSIC y presta especial atención a cuestiones de futuro de esta disciplina en el marco de esta institución y a escala nacional.

\section{ANTECEDENTES HISTÓRICOS}

En 1953 el Consejo Superior de Investigaciones Científicas creó el Centro de Información y Documentación (CID) del Patronato Juan de la Cierva. La creación de este organismo significó la formalización de unas actividades que se venían desarrollando en el Patronato Juan de la Cierva, en el marco de la Sección Extranjera de su Secretaría General, que organizaba la documentación que se suministraba a los Institutos y al personal directivo del Patronato.

En el CID se estableció el Servicio de Consultas Bibliográficas y se inició la publicación del Índice de Revistas Científicas y Técnicas, que sería más tarde sustituido por los Resúmenes de Artículos Científicos y Técnicos.

En la década de los 70, y como consecuencia de un informe de la OCDE sobre la política española en materia de información y documentación titulado Examens des politiques nationales de l'information scientifique et technique. Espagne (OCDE, 1974), se crea un órgano coordinador del Plan Nacional de Información Científica y Técnica como rector de todas estas actividades, con el nombre de Centro Nacional de Información y Documentación Científica (CENIDOC). EI CENIDOC coordinaba tres Institutos orientados sobre grandes áreas del conocimiento: Ciencia y Tecnología, Biomedicina y Ciencias Sociales y Humanidades. De esta forma, en 1975, el CID se convierte en el Instituto de Información y Documentación en Ciencia y Tecnología (ICYT). En el mismo año, se crea, en el CSIC, el Instituto de Información y Documentación en Ciencias Sociales y Humanidades (ISOC), a partir del Departamento de Información Científica y Técnica del Instituto Bibliográfico Hispánico del Ministerio de Cultura. De igual forma se crea el de Biomedicina, sobre el Centro de Documentación e Informática Médica de Valencia. La actuación de estos tres Institutos se basaba en tres elementos fundamentales: investigación, docencia y servicios en el área de la Información y la Documentación Científica.

En realidad, el CENIDOC vino a ser un exponente pionero en materia de política de información científica, sin embargo no llegó a cumplir su objetivo como ente coordinador de los servicios y centros de documentación científica existentes entonces.

En 1975 se instalaron en el ICYT y en el ISOC los primeros terminales para el acceso en línea a grandes distribuidores de información: DIALOG, QUESTEL, ORBIT, etc., constituyéndose así en pioneros en el uso, en España, de las redes telemáticas y las grandes bases de datos para fines científicos.

EI ISOC publica por primera vez, en 1976 , el Índice Español de Humanidades y el Índice Español de Ciencias Sociales. El ICYT inicia, en 1979, la edición del Índice Español de Ciencia y Tecnología. Estos tres Índices recogen, en forma de referencia bibliográfica, los artículos publicados en las revistas científicas españolas de sus respectivas áreas y darán lugar a las Bases de Datos ISOC e ICYT respectivamente.

En 1989 las Bases de Datos ICYT, de bibliografía en Ciencia y Tecnología, e ISOC, de bibliografía en Ciencias Sociales y Humanidades, empiezan a distribuirse en línea desde el Centro Técnico de Informática del CSIC. Las Bases de Datos del CSIC (que además de las anteriores cuentan con el Índice Médico Español, base de datos producida conjuntamente por el CSIC y la Universidad de Valencia) fueron, en 1990, el primer producto de información bibliográfica editado en España en soporte CD-ROM.

\section{EL CENTRO DE INFORMACIÓN Y DOCUMENTACIÓN CIENTÍFICA}

En 1992 se creó el Centro de Información y Documentación Científica (CINDOC) como resultado de 
la fusión del Instituto de Información y Documentación en Ciencia y Tecnología (ICYT) y el Instituto de Información y Documentación en Ciencias Sociales y Humanidades (ISOC) asumiendo de forma integradora los objetivos y funciones de ambos para potenciar la información científica de alta calidad en todos los campos del conocimiento, a excepción del de medicina, cubierto por el Instituto de Historia de la Ciencia y Documentación López Piñero.

EI CINDOC constituía en sí mismo, y por la variedad de actividades e interrelaciones científicas, técnicas, académicas y de asesoría en las que estaba comprometido, algo más que el germen de un futuro sistema de información científica para el CSIC, ya que además de estar considerado como un centro nacional de información y documentación, albergaba una gran biblioteca multidisciplinar y dos bibliotecas especializadas, así como la mayor hemeroteca científica del país, disponía de un departamento de acceso y consulta a prácticamente la totalidad de las bases de datos bibliográficas accesibles on-line, disponía de un servicio de acceso al documento primario, realizaba numerosas actividades de docencia especializada en el ámbito de la información y documentación y el elevado nivel de ingresos que generaba al CSIC en base a la prestación de servicios constituía un fiel reflejo de su utilidad, no solo para investigadores del CSIC sino para multitud de usuarios externos. Muchos de estos usuarios y buena parte de la captación de fondos obedecían a los servicios y utilidades proporcionadas por las bases de datos ICYT e ISOC. Asimismo, este Centro desarrollaba una serie de actividades de interés clave para el desarrollo y normalización de múltiples actividades en el marco de la información y documentación científica, tales como el apoyo y asesoramiento a editores españoles de revistas científicas, el eficaz apoyo prestado al incremento de la difusión internacional de las revistas científicas españolas, las interacciones con AENOR y con otras instituciones españolas y extranjeras (FID, UNESCO, etc.) implicadas, o con competencias, en el avance de un ámbito disciplinar que en España se encuentra todavía en clara situación de desfase respecto a otros países científica y tecnológicamente mejor situados. Por si todo lo anterior no fuera suficientemente importante para el CSIC, y para el conjunto de la comunidad científica española, el CINDOC generó varios grupos de investigación que desempeñaban su labor en distintos frentes de análisis de la ciencia y la tecnología. Estos grupos, como consecuencia de políticas institucionales, se verían seriamente reducidos en cuanto al número de investigadores, y pasaron a conformar el Instituto de Estudios Documentales sobre Ciencia y Tecnología (IEDCYT). En el esquema de funcionamiento del CINDOC las interacciones entre investigadores y documentalistas fueron extremadamente fructíferas para dichos colectivos y en especial para el incremento cuantitativo y cualitativo de las actividades desempeñadas por ambos.
Así el CINDOC ha venido realizando una doble misión; por una parte como centro del CSIC dedicado a información y documentación científica, del que ha sido un referente a escala nacional e internacional $y$, por otra, como un importante núcleo donde se han generado diversos grupos de investigación dedicados fundamentalmente a estudios sobre ciencia y tecnología y sobre la transferencia e impacto de la investigación científica en la sociedad.

En 2007, se crea el Centro de Ciencias Humanas y Sociales (CCHS) del CSIC, del que pasaron a formar parte siete Institutos, uno de ellos el Instituto de Estudios Documentales sobre Ciencia y Tecnología (IEDCYT), que desde ese momento abandona su anterior denominación (CINDOC) y adquiere la misión y objetivos propios y singulares de un instituto de investigación específica y únicamente orientado a realizar estudios sobre la ciencia y la tecnología.

Las unidades de carácter técnico documental del CINDOC; Bases de datos ICYT e ISOC, la Biblioteca (que incluía la mayor hemeroteca científica de España) y las unidades de Reprografía y Gerencia y Servicios administrativos, siguen distintas trayectorias que culminan con la desaparición de buena parte de ellas. Actualmente solo perduran las bases de datos ISOC e ICYT, como una única Unidad dependiente del CCHS, mientras que la Biblioteca del antiguo CINDOC ha sido desmantelada y sus fondos documentales transferidos a otras bibliotecas de diferentes institutos y centros del CSIC, y en gran medida a la Biblioteca Tomás Navarro Tomás de humanidades y ciencias sociales del CCHS. Asimismo, se suprimieron las suscripciones del CINDOC a numerosas revistas científicas, prácticamente todas las de ciencias experimentales y dispersado o eliminado el resto de los fondos correspondientes a disciplinas de ciencia y tecnología.

Este proceso culmina con la desaparición del CINDOC, lo que responde a una decisión que, no siendo en ningún momento argumentada públicamente por las entonces autoridades del CSIC, tampoco tuvo en cuenta las recomendaciones de la evaluación externa (realizada por iniciativa del CSIC) sobre la Propuesta de Plan Estratégico del CINDOC en fechas anteriores a la creación del CCHS. Dicha evaluación externa dio como resultado un informe favorable al CINDOC y a su propuesta estratégica. Resulta significativo que en ningún momento se siguió el protocolo que especifica el Reglamento del CSIC para la supresión de centros o institutos, sino que se optó por "cambiar de denominación" al CINDOC, transformándolo en el Instituto de Estudios Documentales sobre Ciencia y Tecnología (IEDCYT), confiriendo a este último una labor estrictamente investigadora en el marco del CCHS, y por ir desmantelando de forma paulatina, pero continuada, casi todas las estructuras funcionales del CINDOC hasta su práctica extinción, desapareciendo así casi todos sus productos y servicios a la 
comunidad científica. Junto a las antes mencionadas bases de datos ICYT e ISOC, sólo perdura la Revista Española de Documentación Científica, que en fechas próximas a la desaparición formal del CINDOC pasó a formar parte de los fondos de revistas de las bases de datos de ISI-Thomson, y ha continuado hasta nuestros días bajo la dirección de personal adscrito al IEDCYT, y actualmente sigue su andadura y se ha consolidado como una revista científica de difusión internacional que da cabida a intereses científicos en el ámbito de la información y documentación y de los estudios sobre la ciencia y la tecnología.

\section{EL SISTEMA DE INFORMACIÓN CIENTÍFICA DEL CSIC}

Paralelamente al proceso de desmantelamiento del CINDOC, el CSIC inicia el proceso de definición y creación del que se denomina Sistema de Información Científica (SIC) del CSIC (CSIC, 2011). Paradójicamente, este Sistema se configura a partir de la anteriormente denominada Unidad de Coordinación de Bibliotecas (actualmente Unidad de Recursos de Información Científica para la Investigación - URICI) y del Departamento de Publicaciones (actualmente Editorial CSIC), quedando fuera de su cobertura las bases de datos ICYT e ISOC y el resto del personal y actividades técnicas que desempeñaba el CINDOC.

La configuración del mencionado SIC del CSIC, y su creación por Resolución del 31 de mayo de 2011 ha sido, sin lugar a dudas, una ocasión perdida para dotar a la Institución de un verdadero sistema de información científica y tecnológica acorde con las necesidades actuales y con una proyección de futuro. Igualmente ha sido una ocasión perdida para haber dotado a la mayor institución científica de España con un sistema de información cimentado en una sólida y elaborada política de información en materia de ciencia y tecnología, al amparo de la cual haber definido las herramientas e instrumentos necesarios para un verdadero sistema de información, mas allá de la simple suma de recursos existentes y de la práctica desaparición de lo mucho que aportaba el CINDOC, incluida su labor de formación de nuevos especialistas en información y documentación científica.

En la Misión encomendada al SIC, el documento de Resolución establece que éste cubrirá, entre otras, la necesidad de "Definir la política institucional sobre Información Científica".

Es particularmente grave que en vez de partir de una política de información científica claramente establecida sobre la base de un riguroso estudio y de la identificación de fortalezas, debilidades, oportunidades y necesidades desde el punto de vista de la planificación estratégica, se haya optado por constituir un sistema de información al que se le encomienda definir la política institucional sobre información científica. Es obvio lo arriesgado de una decisión de esta naturaleza, ya que, de llevarse a cabo esta encomienda, se corre el riesgo de que lo propuesto responda más a las capacidades y funciones de las distintas unidades y estructuras preexistentes y no responda a un proceso de análisis e identificación de futuras necesidades y desafíos para un SIC que debería gestarse como un elemento transversal al CSIC, pero con una misión y unos objetivos mucho mas ambiciosos y acordes con las necesidades del propio CSIC y con la realidad científica, económica y social del país.

Curiosamente, la mencionada Resolución, en el apartado de Misión, establece también que el SIC cubrirá la necesidad de "Coordinar eficientemente todas las unidades y departamentos con parcelas de competencia en información científica, publicaciones y divulgación de la ciencia."

Resulta particularmente llamativo que ni entre las actuaciones previstas ni en las necesidades que ha de cubrir el SIC figure la creación de ninguna nueva unidad o estructura, limitándose el Sistema a operar con las unidades preexistentes a fecha de la Resolución. Es más, la Resolución señala explícitamente en el apartado dedicado al Esquema organizativo, que "El modelo propuesto parte de unidades existentes, no supone una agregación o una fusión, siendo fundamentalmente un esquema relacional para coordinar mejor las unidades, crear sinergias y optimizar sus recursos, tanto humanos como tecnológicos".

Resulta a este respecto bastante desalentador comprobar cómo la desaparición del CINDOC ha provocado, junto a la desaparición de muchas funciones que éste desempeñaba, una dispersión de personal especializado y experimentado en tareas de información y documentación científica hacia destinos y funciones de otra naturaleza, y cómo en ciertos casos algunos especialistas han optado por su marcha a otras instituciones. Resulta preocupante el hecho de que las bases de datos bibliográficas ICYT e ISOC hayan quedado excluidas del actual SIC del CSIC y que aun a fecha de hoy perduren ciertas incertidumbres sobre el futuro de las mismas y sobre el valor que la institución le otorga a éstas.

Igualmente preocupante es la evolución de la base de datos Índice Médico Español, que, como ya se ha señalado, ha venido siendo producida conjuntamente por la Universidad de Valencia y el CSIC y que en la actualidad se encuentra comprometida en un proceso en el que se pretende su transferencia total o parcial al Instituto de Salud Carlos III.

El esquema organizativo propuesto para el SIC del CSIC, además de partir de unidades preexistentes "se concibe como una red de tres subsistemas para la gestión de la información científica coordinados por una Comisión de Política de Información Científica". Según la Resolución, la Comisión estará presidida por el Vicepresidente 
de Organización y Relaciones Institucionales del CSIC y compuesta por los titulares de varias Vicepresidencias Adjuntas, el titular de la Secretaría General Adjunta de Informática, el responsable de la Unidad de Coordinación de Bibliotecas (a partir de ese momento "Recursos de información científica para la investigación") el director del Departamento de Publicaciones y dos miembros del personal científico del CSIC designados por el Vicepresidente de Investigación Científica y Tecnológica.

El esquema organizativo planteado en la Resolución, hace así que las responsabilidades recaigan sobre altos cargos del CSIC y responsables de unidades que tienen encomendadas unas funciones específicas en el conjunto del sistema, pero puede resultar deficitario desde el punto de vista práctico, ya que no designa un responsable operativo que actúe de forma resolutiva ante los imprevistos y las interacciones que regularmente se efectúan entre los diversos usuarios y agentes del ámbito de la información y documentación científica (tanto nacionales como extranjeros) y el CSIC. Resulta evidente que, pese al estudio encargado por el CSIC a una consultora privada para ayudar a fundamentar el nuevo SIC, el desconocimiento de la realidad del día a día, en este sector de actividad, ha dejado su huella a la hora de planificar dicho esquema organizativo.

Por todo lo anterior, cabe entender que las actuaciones descritas, lejos de beneficiar al CSIC, han causado enormes perjuicios a la institución y a los múltiples usuarios internos y externos del antiguo CINDOC. El periodo de tiempo transcurrido desde octubre de 2007, momento en el que se crea el CCHS y el CINDOC desaparece como Centro de Información y Documentación Científica, hasta la fecha de hoy, resulta escasamente productivo para el ámbito de la información y documentación, así como desarticulador de las eficaces dinámicas preexistentes. Parece, por tanto, más que justificado plantear la revisión del modelo vigente en aras de dotar a la institución, y al conjunto de la comunidad científica española, de un Sistema Integrado de Información Científica que, como ya se ha señalado en este documento, tenga por cimientos una clara y definida política de información científica y cuente con un esquema organizativo y de dirección claramente definido y con capacidad operativa.

\section{LOGROS RECIENTES}

Pese a lo anterior, durante los últimos años se han puesto en marcha en el CSIC algunas iniciativas en el ámbito de la información y documentación científica. Iniciativas que, pese a sus logros, no responden a una planificación institucional en esta materia. Entre éstas cabe destacar las siguientes: Digital.CSIC, que es el repositorio institucional del Consejo Superior de Investigaciones Científicas. Éste es un depósito de documentos en formato digital, cuya finalidad es la de organizar, archivar, preservar y difundir en acceso abierto la producción intelectual que es resultado de las actividades investigadoras del CSIC. Dicho proyecto, desarrollado por la Red de Bibliotecas del CSIC, se inició en enero de 2008 y se fundamenta en la firma por parte de la Presidencia del CSIC de la Declaración de Berlín en 2006 (Berlin Declaration on Open Access to Knowledge in the Sciences and Humanities, 2003): dicha Declaración es uno de los hitos fundacionales del movimiento internacional del acceso abierto que ha servido de estímulo a los centros europeos de investigación para tratar de dar la mayor visibilidad e impacto posible a los resultados de la investigación mediante la creación y puesta a punto de archivos institucionales en abierto. El objetivo principal de Digital CSIC es el de ser una ventana que aumente la visibilidad y el impacto de la investigación científica del CSIC.

La Plataforma Open Access de Revistas Científicas Electrónicas Españolas y Latinoamericanas, e-Revistas, es un proyecto impulsado por el CSIC con la finalidad de contribuir a la difusión y visibilidad de las revistas científicas publicadas en países de América Latina y del Caribe, así como de Portugal y España. El proyecto se inició en 2004 como parte del Portal Tecnociencia, creado en el marco de un convenio FECYT-CSIC (2004-2006). Hasta 2009 e-Revistas se ha venido desarrollando en el Instituto de Estudios Documentales sobre Ciencia y Tecnología (IEDCYT-CSIC) con financiación a cargo de proyectos intramurales. En la actualidad e-Revistas se desarrolla en Editorial CSIC, Departamento que posee la Certificación de Calidad conforme a la norma ISO 9001 (ER-0285/2009).

El objetivo del portal e-Revistas es el de reunir, seleccionar y difundir las revistas científicas españolas y latinoamericanas, de cualquier disciplina científica y en cualquier idioma, que se publiquen en acceso abierto. La plataforma e-Revistas ofrece libre acceso al texto completo de los contenidos de las revistas que se incorporan a la misma. No obstante, se admite un periodo de embargo del último número publicado que, siguiendo las recomendaciones de la Unión Europea, no supera los seis meses. Rigiéndose por los criterios de calidad Latindex para revistas electrónicas, las revistas que participan en este proyecto deben satisfacer unos estándares de calidad formal y de contenido.

El portal e-Revistas actúa como proveedor de datos conforme al protocolo OAI-PMH (Open Archives Initiative - Protocol for Metadata Harvesting) permitiendo así que otros portales, bases de datos y servicios de información puedan acceder a los metadatos de los documentos publicados.

\section{ACTIVIDAD EDITORIAL}

El Consejo Superior de Investigaciones Científicas, inició su actividad editorial en el mismo año de su creación, 1939. Actualmente la Editorial 
CSIC, cuenta con un catálogo constituido por más de 2200 libros (que cubren todas las disciplinas científicas) y 41 revistas científicas, que figuran en las mas importantes bases de datos bibliográficas internacionales. Actualmente en la Web of Science hay indizadas 318 revistas españolas, de las cuales 21 son editadas por el CSIC. En Scopus hay 584 revistas españolas, de las que 28 pertenecen al Consejo. Editorial CSIC trata de difundir resultados de investigación en temas de relevancia social, divulgación científica de alto nivel y sobre conmemoraciones científicas y culturales. En 2013 su fondo de libros se ha visto incrementado en 66 nuevos títulos.

La edición de revistas científicas por parte de diversos institutos del CSIC es otro frente de actuación que merece ser tenido en cuenta a la hora de describir el panorama actual de la información y documentación científica en el CSIC. Actualmente el número de títulos de revista (vigentes) producidos por la institución es 40 (37 de ellas en formato electrónico) contando con la reciente incorporación en 2012 de una nueva revista científica Culture and History Digital Journal, producida por el Instituto de Historia del CSIC. Durante los últimos años, los editores de estas publicaciones periódicas y Editorial CSIC han hecho un importante esfuerzo de mejora de las mismas, de tal manera que se ha producido un claro incremento del impacto y difusión internacional de estas revistas a través del acceso electrónico abierto en la Plataforma Revistas Científicas del CSIC (http://revistas.csic.es).

\section{HACIA UNA ESTRATEGIA DE FUTURO}

El actual panorama económico y social en el que se enmarca el sistema científico español plantea no pocas dificultades y retos que requieren, no solo un decidido y continuado esfuerzo por parte de los agentes implicados en I+D para superar las consecuencias de una creciente disminución de recursos, sino la puesta en práctica de actuaciones innovadoras y acordes con la situación actual y con las necesidades futuras. En este contexto, el CSIC debe contribuir eficazmente a una renovación de la política científica en España. Renovación que implica para esta institución asumir una serie retos que hagan que siga siendo un referente científico indiscutible a escala nacional e internacional, alcance mayores y más importantes metas científicas y logre una más eficaz interacción con los agentes políticos, sociales y con los sectores productivos de la sociedad.

Es importante que pese a lo adverso de la actual coyuntura, el CSIC considere los años venideros como una oportunidad para poner en valor los conocimientos científicos generados en etapas recientes, así como las experiencias investigadoras y en materia de gestión. Sería un objetivo deseable para estos próximos años incrementar significativamente la visibilidad de la institución y la oferta de conocimientos y know-how hacia los sectores productivos, fundamentalmente hacia aquéllos que pueden ser esenciales en la recuperación económica y social del país. Dada la situación económica actual, sería esencial para el CSIC mantener en un nivel adecuado las capacidades y actividades investigadoras en aquellos ámbitos disciplinares con mayor potencial de transferencia de conocimientos a los sectores productivos, especialmente hacia aquéllos que se han visto más perjudicados por la situación de crisis económica y hacia aquéllos que más pueden aportar a la recuperación.

Asimismo, las limitaciones presupuestarias actuales deberían llevar a considerar este momento como una oportunidad para llevar a cabo una serie de iniciativas estratégicas que no requieran un elevado coste económico o que puedan ser realizadas de forma progresiva de tal manera que se ajusten a una planificación que las haga económicamente viables.

Tal vez este sea un buen momento para que el CSIC efectúe un ejercicio de reflexión sobre el valor estratégico de la información científica y sobre sus actuales capacidades y limitaciones en materia de información y documentación y en cómo este ámbito disciplinar puede contribuir a la misión y a los objetivos generales de la institución.

A tenor de lo anteriormente expuesto, queda fuera de duda la conveniencia de definir y poner en práctica una Política de información científica y tecnológica propia para el CSIC. Una decisión acorde requeriría un análisis objetivo de la situación actual, de las estrategias institucionales y de las perspectivas de futuro, así como aprender de los aciertos y errores del pasado. Sería también aconsejable un análisis de las principales estrategias en materia de información y documentación llevadas a cabo por instituciones extranjeras semejantes al CSIC, como es el caso del CNRS. Esta institución otorga al ámbito de la información científica un papel predominante en su esquema organizativo y operativo (CNRS, 2013). De hecho, el CNRS cuenta con una Dirección General de Información Científica y Tecnológica (Direction de I'information scientifique et technique - DIST) (http://www.cnrs. $\mathrm{fr} / \mathrm{dist} /$ ) entidad que puede considerarse con rango equiparable al de una Vicepresidencia Adjunta del CSIC, pero que en este caso desempeña además un papel clave en la comunidad de información y documentación científica francesa (CNRS, 2011). En este contexto, juega un papel clave el INIST (Institut de l'information scientifique et technique) (http://www.inist.fr/?lang=fr), un instituto propio del CNRS que ya cuenta con 25 años de historia y muy similar en sus funciones a lo que era el CINDOC del CSIC.

Por no hacer una lista extensa, cabe citar como ejemplo algunas otras entidades de $I+D$, de reconocido prestigio, que cuentan con políticas de información y documentación científica y de los instrumentos y herramientas apropiadas, como es el caso del Max Planck Gessellschaft que dispone, 
entre otros, de unos Servicios de Recuperación de Información y de una Biblioteca Digital (MPG), el CERN Scientific Information Service de la Organización Europea para la Investigación Nuclear (CERN) (http://oldlibrary.web.cern.ch/oldlibrary/ WhoWeAre/Mission.html), el Center for Science of Information de la National Science Foundation (NSF) (http://soihub.org/about-us.php) o el Institute for Scientific and Technical Information del National Research Council de Canadá (NRCC) (http:// cisti-icist.nrc-cnrc.gc.ca/eng/ibp/cisti/index.html).

Una clara Política de información en ciencia y tecnología para el CSIC sería la base conceptual sobre la que podría constituirse un Sistema integrado de información científica, y lo que permitiría definir su plan estratégico de actuación y las unidades, servicios, herramientas de información, utilidades e interrelaciones.

Un nuevo sistema de información para el CSIC debería, no solo ser capaz de movilizar adecuadamente los recursos y herramientas de información actualmente disponibles en la institución, sino de transformar aquéllas que sean necesarias y crear y coordinar nuevos elementos, incluso con objetivos estrictamente investigadores. Bajo estas premisas, constituiría un decisivo avance cualitativo sobre otros modelos o pretensiones más cercanas a la simple agregación de unidades y servicios preexistentes (y fuertemente mermados) en el CSIC.

En síntesis, una política de información científica para el CSIC y su correspondiente sistema de información, darían respuesta a la necesidad de dotar a la institución con un sistema de recursos y herramientas de información con valores añadidos y con un significativo poder de interrelación que permitiría:

1. Satisfacer las demandas de información científica y tecnológica a diferentes tipos de usuarios y necesidades.

2. Servir de elemento clave en la visibilidad y proyección nacional e internacional del CSIC en distintas esferas y sectores sociales, económicos y políticos.

3. Contribuir a incrementar y mejorar la percepción social de la investigación científica y sus agentes.

4. Contribuir a las labores de análisis de información, vigilancia e inteligencia estratégica y planificación científica.

Una última reflexión sobre el posible establecimiento de una política de información científica en el CSIC y un sistema de información acorde con una misión y unos objetivos claramente definidos, obedece a la conveniencia de que esta institución alcance, en el contexto nacional, una situación de liderazgo y se sitúe en una posición estratégica en materia de política y planificación de actividades de información en ciencia y tecnología. No conviene olvidar que en la actualmente vigente Ley 14/2011 de la Ciencia la Tecnología y la Innovación (España, BOE, 131, 2 de junio de 2011) se formula como objetivo en su Artículo 11, crear un "Sistema de información sobre ciencia, tecnología e innovación".

En nuestro país no se ha realizado un esfuerzo significativo en materia de políticas de información y documentación (Gómez Fernández-Cabrera, 1999). En la actualidad, España posee un cierto número de centros de documentación especializada, pero no puede hablarse de una política estructurada en materia de información y documentación científica. El único intento realmente destacable en esta materia se remonta a 1982, momento en el que el Ministerio de Educación y Ciencia creó la Subdirección General de Documentación e Información Científica, dependiente de la Dirección General de Política Científica. Esta Subdirección estableció un Plan de actuación en materia de Documentación e Información Científica y Técnica (Plan IDOC) y entre 1983 y 1986, al amparo de dicho Plan se elaboraron una serie de estudios sobre la situación en el sector. Más allá de los correspondientes informes y recomendaciones, el Plan carecía de un marco legislativo que le permitiera desarrollarse $y$, por tanto, tampoco contaba con una financiación que hiciera posible avanzar en las propuestas realizadas por los expertos que trabajaron en aquella iniciativa.

EI CSIC posee una experiencia demostrada en materia de información y documentación científica; sin embargo la historia reciente nos muestra que la institución ha estado lejos de mejorar y coordinar eficazmente los productos, herramientas y recursos técnicos y humanos en este ámbito, e incluso de conservar muchos de éstos. Siendo la información científica y tecnológica un bien de indiscutible y creciente valor estratégico, urge llevar a cabo las iniciativas y estrategias necesarias para situar a este ámbito disciplinar en la posición que le corresponde en el CSIC, contribuyendo así al logro de los objetivos generales y estratégicos de la institución.

\section{BIBLIOGRAFÍA}

Berlin Declaration on Open Access to Knowledge in the Sciences and Humanities (2003). http:// oa.mpg.de/files/2010/04/berlin declaration.pdf [Consulta: 11:10:2013].

CNRS (2011). Étude sur les pratiques en Information scientifique et technique au CNRS http:// www.dgdr.cnrs.fr/drh/omes/documents/pdf/RapportIST_2011.pdf [Consulta: 11:10:2013].

CNRS (2011). Direction de I'information scientifique et technique. http://www.cnrs.fr/dist/ [Consulta:11:10:2013].

CNRS (2013). Institut de l'information scientifique et technique INIST. http://www.inist.fr/?lang =fr [Consulta 16:10:2013].

CSIC (2011). Resolución del Presidente del Consejo Superior de Investigaciones Científicas por 
la que se estructura el Sistema de Información Científica (SIC). http://www.ebd.csic.es/website1/Novedades/Documentos/C2BC6d01.pdf [Consulta: 11:10:2013].

CERN Scientific Information Service http://oldlibrary.web.cern.ch/oldlibrary/WhoWeAre/Mission.html [Consulta: 11:10:2013].

España. Ley 14/2011 de 1 de junio de la ciencia, la tecnología y la innovación. BOE, 131. http:// www.boe.es/boe/dias/2011/06/02/pdfs/BOEA-2011-9617.pdf [Consulta: 11:10:2013].

Gómez Fernández-Cabrera, J. (1999): Políticas de Información y Documentación en España.- En GARCIA GUTIERREZ, A.(ed.), Introducción a la Documentación Informativa y Periodística. Sevilla: Editorial Mad, pp.155-185. ISBN 848311-460-7.

MPG Max Plank Gessellschaft Information Retrieval Services http://www.mpg.de/807741/
Information Retrieval_Services [Consulta: 16:10:2013].

NRCC National Research Council Canada - Institute for Scientific and Technical Information (NRCCISTI): http://cisti-icist.nrc-cnrc.gc.ca/eng/ibp/ cisti/index.html [Consulta: 16:10:2013].

NSF National Science Foundation - Center for Science of Information http://soihub.org/aboutus.php [Consulta: 16:10:2013].

OCDE (1974). Examens des politiques nationales de l'information scientifique et techniques: Espagne.

Zins, C. (2007). Classification schemes of Information Science: Twenty Eight Scholars maps the field. Journal of the American Society for Information Science and Technology 58(5), 645-672. http://dx.doi.org/10.1002/asi.20506 